described. CSE was prepared by combusting 1 Marlboro cigarette through $25 \mathrm{ml}$ of media. Cell viability was determined after primary nasal epithelial cells (PNECs) were stimulated with 5\% CSE for $24 \mathrm{~h}$ (caspase 3 levels determined after $4 \mathrm{~h}$ ), in the presence or absence of $20 \mathrm{mM} \mathrm{N}$-acetylcysteine (NAC). In separate experiments, cultures were stimulated with Pseudomonas aeruginosa lipopolysaccharide (PA LPS) for $24 \mathrm{~h}(0-30 \mu \mathrm{g} / \mathrm{ml})$, and the effects of pre-incubation with CSE $\pm 20 \mathrm{mM}$ NAC for $4 \mathrm{~h}$ evaluated in terms of cytokine release. Phospho-NF- $\kappa$ B activity was determined after $1 \mathrm{~h}$ PA LPS exposure. Apoptosis was evaluated using annexin-V staining and the terminal transferase-mediated dUTP nick end-labelling (TUNEL) method.

Results $5 \%$ CSE (4 h) exposure was immunosuppressive in PNEC cultures for both IL-8 and IL-6 release (0.53 fold reduction in IL-8 and 0.49 fold reduction in IL- 6 release after stimulation with $30 \mu \mathrm{g} /$ $\mathrm{ml}$ PA LPS for $24 \mathrm{~h}$ ). $4 \mathrm{~h}$ exposure to CSE heightened active caspase 3 levels, and a $24 \mathrm{~h}$ exposure induced both early and late apoptosis established by annexin-V staining (Table 1). Apoptosis was confirmed using the TUNEL assay. All of these effects were mitigated with the addition of 20 mM NAC to the CSE ( 0.85 fold reduction in IL- 8 and 0.73 fold reduction in IL-6 release after stimulation with $30 \mu \mathrm{g} / \mathrm{ml}$ PA LPS for $24 \mathrm{~h}$ ).

Conclusions A $4 \mathrm{~h}$ CSE exposure was immunosuppressive in PNEC cultures and induced apoptosis. Reactive oxidative species are at least partially responsible for these observations.

Research funded by NI RDO.

Abstract P251 Table 1 Annexin-V analysis of 5\% CSE \pm 20 mM NAC treatment in PNEC Cultures.

\begin{tabular}{lcccc}
\hline & Viable & Early Apoptotic & Late Apoptotic & Necrotic \\
\hline Control 24 h & $99.4 \%$ & $0.4 \%$ & $0.1 \%$ & $0.1 \%$ \\
$5 \%$ CSE 24 h & $29.1 \%$ & $47.7 \%$ & $21.2 \%$ & $2.0 \%$ \\
NAC \& 5\% CSE 24 h & $74.0 \%$ & $18.3 \%$ & $4.8 \%$ & $2.9 \%$ \\
\hline
\end{tabular}

\section{P252 PD-1 EXPRESSION ON HUMAN LUNG T CELLS IN HEALTH AND COPD}

doi:10.1136/thoraxjnl-2012-202678.344

RT McKendry, CM Spalluto, KJ Staples, TMA Wilkinson. The University of Southampton, Southampton, United Kingdom

Introduction and Objectives Patient $\mathrm{s}$ with chronic obstructive pulmonary disease (COPD) are susceptible to the effects of recurrent respiratory infections despite increased numbers of $\mathrm{CD} 8+\mathrm{T}$ cells in the lungs. We hypothesised that the inability of $\mathrm{CD} 8+\mathrm{T}$ cells to successfully combat respiratory pathogens in COPD may be due to T cell "exhaustion" - a phenomenon described in chronic infections. Exhausted CD8+ T cells have significantly reduced cytotoxicity and inflammatory cytokine release. Exhaustion is thought to be initiated by the binding of PD-1 on T cells to its ligand (PD-L1) which is expressed on epithelial cells and macrophages. PD-1 expression is upregulated in murine models of acute and chronic viral infection, but this has yet to be elucidated in human cells.

We aimed to identify and quantify PD-1+CD4+ and CD8+ T cells and cells expressing PD-L1 in the lungs of COPD patients and non-COPD controls.

Methods Lung tissue from patients undergoing surgery was digested using collagenase to form single-cell suspensions. Lung $\mathrm{T}$ cells were identified as populations of $\mathrm{CD} 45+\mathrm{CD} 3+$ cells which were either CD4+CD8- or CD4-CD8+. T cells expressing PD-1 were quantified by multi-colour flow cytometry. Patients with a FEV1/FVC ratio $<70 \%$ were defined as having COPD.

Results The proportion of CD8 $+\mathrm{T}$ cells in the COPD lung (mean expression $=40.87 \%, \mathrm{SD}=14.67)$ was significantly higher $(\mathrm{p}=0.013$, students t-test) than in non-COPD (mean expression $=26.74 \%$, $\mathrm{SD}=11.12$ ), reflecting previous findings. $\mathrm{PD}-1$ expression in $\mathrm{CD} 4+$ $T$ cells appeared to be lower in COPD (mean expression $=39.91 \%$, $\mathrm{SD}=13.02$ ) than non-COPD (mean expression $=50.53 \%$, sd $=13.05$ ) but this was not significant. PD-1 expressing CD4+ cells (mean expression $=2.17 \%, \mathrm{SD}=1.4$ ) and $\mathrm{CD} 8+$ cells (mean expression $=6.02 \%, s d=5.73$ ) were detected in tissue, but not in the blood of the same patients. PD-L1 was undetectable on lung epithelial cells but was expressed on macrophages (mean expression $=2.85 \%$, $\mathrm{SD}=1.91)$.

Conclusion Elements of the exhaustion pathway are expressed in the human lung in stable COPD. Further work is needed to clarify if there is an upregulation of this pathway in COPD that may explain the susceptibility of these patients to viral exacerbation. Exhaustion of cells recognising respiratory pathogens may have a significant role in COPD outcomes and requires further elucidation.

\section{P253 ASSOCIATION OF DEFECTIVE MONOCYTE-DERIVED MACROPHAGE PHAGOCYTOSIS WITH CLINICAL PHENOTYPES IN STABLE COPD}

doi:10.1136/thoraxjnl-2012-202678.345

${ }^{1} \mathrm{R}$ Singh, ${ }^{2} \mathrm{KK}$ Chana, ${ }^{3} \mathrm{LL}$ Wright, ${ }^{1} \mathrm{DS}$ Garcha, 'BS Kowlessar, ${ }^{1} \mathrm{ARC}$ Patel, ${ }^{1} \mathrm{AJ}$ Mackay, 2PS Fenwick, 'GC Donaldson, ${ }^{2} \mathrm{PJ}$ Barnes, 'JA Wedzicha, ${ }^{2}$ LE Donnelly. ${ }^{1}$ Centre for Respiratory Medicine, UCL, London, UK; ${ }^{2}$ Airway Disease Section, NHLI, Imperial College London, London, UK; ${ }^{3}$ Centre for Clinical Microbiology, UCL, London, UK

Introduction Macrophages play an important role in clearing inhaled particles and bacteria from the lung, thus maintaining its sterility. Defective phagocytosis of bacteria has been demonstrated in both alveolar and monocyte-derived macrophages (MDMs) from COPD patients and may play a role in the aetiology of the frequent exacerbator phenotype. We hypothesised that defective phagocytosis may also be associated with lower airway bacterial colonisation (LABC) and other clinical parameters in stable COPD

Methods Whole blood and sputa were collected from stable patients in the London COPD cohort. Stable COPD was defined as no symptom-defined exacerbations recorded on prospectively completed diary cards in the preceding four weeks and subsequent two weeks. Monocytes were isolated from the whole blood and cultured with GM-CSF (2 ng/ml) for 12 days to generate MDMs. MDM phagocytosis of fluorescently-labelled polystyrene beads, Haemophilus influenzae (HI) and Streptococcus pneumoniae (SP) was measured by fluorimetry. LABC was defined as detection of HI, SP or Moraxella catarrhalis $(\mathrm{MC})$ in sputum using quantitative PCR.

Results MDMs were cultured from 26 COPD patients. 54\% were male, mean age 70.0 years (SD 8.3), $\mathrm{FEV}_{1}$ predicted $55.3 \%$ (20.3), $46 \%$ were current smokers, median daily inhaled corticosteroid (ICS) dose was 1000 (640-2000) mcg (beclomethasone equivalent dose) and median exacerbation frequency per year was 1.8 (1.0-2.9) based on diary card events.

Phagocytosis of HI was significantly less with increasing exacerbation frequency $(p=0.002, r=-0.58$, Figure 1$)$, although no significant associations were demonstrated between exacerbation frequency and phagocytosis of inert beads or SP $(p=0.27$ and $p=0.22$ respectively). 13 patients (50\%) with LABC did not demonstrate any significant difference in phagocytosis of either beads $(p=0.29)$, HI $(p=0.66)$ or SP $(p=0.88)$ compared with non-colonised patients. There was no significant association between phagocytosis of beads, $\mathrm{HI}$ or SP with age, FEV \% predicted, smoking pack year history, ICS dose or BMI (all $p>0.05$ )

Conclusion In stable COPD patients, decreasing phagocytosis of $\mathrm{HI}$ was associated with increasing exacerbation frequency. Phagocytosis was not related to LABC suggesting that macrophage activity alone may not determine bacteria colonisation. Further work is needed to elucidate the mechanisms of reduced phagocytosis in COPD and its relationship to exacerbation frequency. 


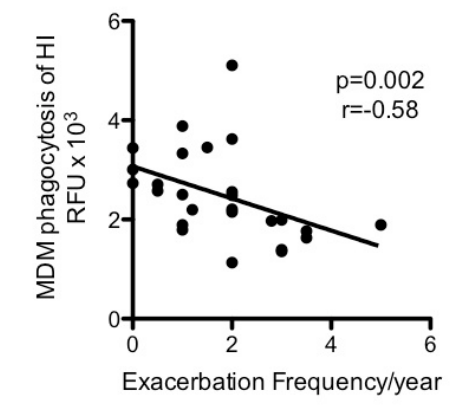

Figure 1. Relationship between MDM phagocytosis of fluorescentlylabelled Haemophilus influenzae and exacerbation frequency.

Abstract P253 Figure 1

\section{P254 IDENTIFYING MMP-12 SUBSTRATES AS THERAPEUTIC TARGETS IN COPD}

doi:10.1136/thoraxjnl-2012-202678.346

'B Mallia-Milanes, 'D Clements, ${ }^{2} \mathrm{~A}$ Sheehan, 'C Bolton, 'SR Johnson. 'University of Nottingham, Nottingham, UK; ${ }^{2}$ Nottingham University Hospitals NHS Trust, Nottingham, UK

Background Matrix metalloprotease (MMP)-12 is a key protease in COPD which cleaves pulmonary extracellular matrix and nonmatrix substrates. Variation in MMP-12 activity affects severity of COPD, yet the mechanism of this, including MMP-12's non-matrix substrates in COPD lungs are unknown. Targeting MMP-12 substrates may lead to the development of drugs for COPD with reduced side effects compared to the broader spectrum MMP inhibitors.

Aims To identify MMP-12 substrates of relevance to COPD and determine how their activity affects disease progression in vitro and in vivo.

Methods In vitro cleavage assays: After literature review the proinflammatory mediators osteopontin and tumour necrosis factor (TNF)- $\alpha$ were selected as potential MMP-12 substrates in COPD. Both were incubated with MMP-12 and reaction products analysed by silver stain and western blot. EDTA was used as a metalloprotease inhibitor and thrombin as positive control. COPD cohort: Patients with COPD were recruited during exacerbations at the Nottingham University Hospitals NHS Trust. Sputum, lung function and other data were collected on Day 0 and 1 and 4 weeks later. Sputum was analysed by western blot for proteins of interest. The study was approved by the local research ethics committee and all patients gave informed consent.

Results MMP-12 cleaved osteopontin and pro-TNF- $\alpha$ in a dose and time-dependent manner when visualised by silver staining. Cleavage was dependent on MMP-12 activity as it was inhibited by EDTA. Western blot of cleaved protein fragments gave a characteristic band signature. MMP-12 was present in sputum of patients with COPD as demonstrated by western blotting, ELISA and casein zymography. Western blot analysis of sputum with anti-osteopontin antibodies showed a similar band signature to the in vitro cleavage suggesting osteopontin is cleaved in the airways of patients with COPD.

Discussion MMP-12 possesses proteolytic activity against osteopontin and pro-TNF- $\alpha$ in vitro. MMP-12, osteopontin and TNF $\alpha$ are present in COPD sputum and our data suggest that MMP-12 may target osteopontin in COPD. Further work is needed to determine the precise mechanisms of such MMP-12 substrate activity in COPD.

\section{P255 THE EFFECTS OF HYPOXIA ON NEUTROPHIL DEGRANULATION}

doi:10.1136/thoraxjnl-2012-202678.347

K Hoenderdos, L Porter, S Alam, N Mc Govern, C Fiddler, J Skepper, ER Chilvers, AM Condliffe. University of Cambridge, Cambridge, Cambridgeshire

Tissues such as the skin and intestinal epithelium experience physiological hypoxia whereas pathological hypoxia occurs at inflammatory sites. Neutrophils are recruited to infective/inflamed areas and are thus required to operate under low oxygen tensions. We have shown previously that hypoxia delays neutrophil apoptosis (JEM 2005; 201:105) and impairs bacterial killing (J Immunol 2011; 186:453) and have now studied the effect of hypoxia on the release of histotoxic neutrophil proteases.

Neutrophils isolated from healthy volunteers were subjected to normoxia or hypoxia $(3 \mathrm{kPa})$. Superoxide anion release was measured by the reduction of cytochrome. C Elastase release was quantified by the cleavage of labelled elastin.

Hypoxic incubation for 4 hours resulted in a 3-fold reduction in superoxide anion release from cells stimulated with GM-CSF and fMLP. In contrast, elastase release from the azurophilic granules was augmented almost 3-fold under hypoxia. The release of MMP-9 and lactoferrin was similarly up-regulated, suggesting a more generalised increase in degranulation under hypoxia. In addition to this electron microscopy showed that hypoxia induced a more activated phenotype (e.g. increased membrane ruffling and cell spreading).

We show that hypoxia can induce a more destructive neutrophil phenotype, with enhanced degranulation and release of potentially histotoxic proteases, impaired bacterial killing, and delayed apoptosis. These data suggest that hypoxia aversively affects neutrophil function and may augment neutrophil mediated tissue destruction.

\section{P256 P2X4 AND NNOS EXPRESSION IN HUMAN CILIATED AIRWAY EPITHELIUM}

doi:10.1136/thoraxjnl-2012-202678.348

I Premadeva, HL Lenartowicz, J Underwood, PM Lackie, JS Lucas, CL Jackson. University of Southampton, Faculty of Medicine, Southampton, UK

Background Calcium $\left(\mathrm{Ca}^{2+}\right)$ and nitric oxide (NO) modulate ciliary beat frequency (CBF). NO synthase (NOS) isoenzymes, responsible for NO production, localise in human airway epithelium and constitutive NOS activity is dependant on $\mathrm{Ca}^{2+}$. Adenosine triphosphate gated purinergic ion channels, comprised of $\mathrm{P}_{2} \mathrm{X}_{1-7}$ subunits, govern $\mathrm{Ca}^{2+}$ influx and $\mathrm{P} 2 \mathrm{X}_{4}$ reportedly localises to rabbit airway cilia. Also, P2X proteins co-localise with neuronal NOS (nNOS) in guinea pig cochlea outer hair cells and rat hypothalamus. We hypothesised that $\mathrm{P}_{2} \mathrm{X}_{4}$ and $\mathrm{nNOS}$ co-localise in human airway cilia and are involved in modulating CBF.

Objectives To determine $P 2 X_{1,3-7}$ mRNA expression and $\mathrm{P} 2 \mathrm{X}_{4}$ and nNOS localisation in human nasal epithelium, and assess their possible interaction in CBF modulation.

Methods and Results Relative to $\beta$-actin expression, consistent and moderate $P 2 X_{4,6}$ mRNA and variable $P 2 X_{3,5,7}$ mRNA were detected by RT-PCR in $(n=4)$ human primary airway epithelium cultured at air-liquid interface (ALI). In ALI cultured primary epithelium $\mathrm{P}_{2} \mathrm{X}_{4}$ localised $(\mathrm{n}=3)$ to cell membranes and cytoplasm and $\mathrm{nNOS}$ localised to cilia $(\mathrm{n}=3)$ by immunofluorescence. P2X localised to the ciliary tips $(n=2)$, whilst nNOS localised to the proximal portion of cilia (specificity confirmed by blocking peptide) in nasal polyp paraffin wax sections by immunohistochemistry $(n=6)$. High speed video microscopy confirmed a 30 minute baseline $\mathrm{CBF}$ at $37^{\circ} \mathrm{C}(12.9 \mathrm{~Hz} \mathrm{SD} \pm 0.8, \mathrm{n}=5)$ on nasal epithelium biopsies 